\title{
Comparison of Gene Expression Profiling and Chromosome 3 Analysis by Fluorescent in situ Hybridization and Multiplex Ligation Probe Amplification in Fine-Needle Aspiration Biopsy Specimens of Uveal Melanoma
}

\author{
Michael A. Klufas ${ }^{a}$ Elizabeth Richter ${ }^{a}$ Sujit ltty ${ }^{\mathrm{a}}$ Christian Moreno ${ }^{\mathrm{a}}$ \\ Colin A. McCannel ${ }^{a, b}$ Tara A. McCannel ${ }^{a, b}$

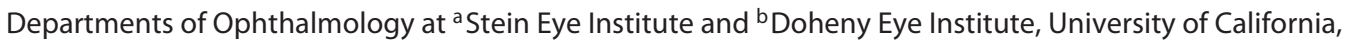 \\ Los Angeles, CA, USA
}

\section{Keywords \\ Uveal melanoma $\cdot$ Choroidal melanoma $\cdot$ Molecular prognostication - Fine-needle aspiration biopsy . Fluorescent in situ hybridization . Gene expression profiling · Multiplex ligation probe amplification . Monosomy 3}

\begin{abstract}
Purpose: The aim of this paper was to assess the concordance between results of DecisionDx-UM specific gene expression profiling (GEP) and fluorescence in situ hybridization (FISH) for chromosome 3 analysis, and between DecisionDx-UM GEP and multiplex ligation probe amplification (MLPA) in uveal melanoma undergoing intraoperative fineneedle aspiration biopsy (FNAB) for metastatic prognostication during brachytherapy. Methods: We retrospectively reviewed consecutive patients diagnosed with posterior uveal melanoma who underwent intraoperative FNAB prior to placement of an iodine- 125 radioactive plaque between 2012 and 2014. Two cohorts of patients were identified: Cohort 1 - tumors in which both GEP and FISH results were ob-
\end{abstract}

\section{KARGER}

(C) 2017 S. Karger AG, Basel

E-Mail karger@karger.com

www.karger.com/oop tained, and Cohort 2 - tumors in which both GEP and MLPA results were obtained. Results: Forty-four patients were identified for Cohort 1. FISH and GEP results were discordant in 7 tumors (15.9\%). Forty-three patients were identified for Cohort 2. MLPA and GEP were discordant in 7 tumors (16.3\%). Conclusions: Discordance between GEP and chromosome 3 status by FISH and MLPA occurred in our series at a rate of 15.9 and $16.3 \%$, respectively. Caution must be advised when counseling a patient with a good-prognosis GEP "Class 1" result that the uveal tumor may actually harbor monosomy 3 , which is associated with a poor prognosis for metastasis in nearly $20 \%$ of the patients.

C 2017 S. Karger AG, Basel

\section{Introduction}

Molecular prognostic biopsy for metastatic risk in the management of patients with uveal melanoma is standard of care at most major ophthalmic oncology practices in North America [1-8]. The loss of 1 copy of chromosome 3 (monosomy $3[\mathrm{M} 3]$ ) in the tumor tissue has been deter-

Tara A. McCannel, MD, PhD

UCLA Stein Eye Institute

100 Stein Plaza

Los Angeles, CA 90095 (USA)

E-Mail TMcCannel@jsei.ucla.edu 
mined to be the risk factor most strongly associated with metastatic death originally reported in mostly large uveal melanoma [9-11]. Today, there are several options for tumor tissue testing to determine metastatic risk, both DNA and RNA based. Among DNA-based tests, fluorescence in situ hybridization (FISH) testing for the centromere region of chromosome 3 was first reported at our center in samples obtained through intraoperative fineneedle aspiration biopsy (FNAB) [12]. Multiplex ligation probe amplification (MLPA) for uveal melanoma prognostic testing was reported in the United Kingdom, and this DNA-based technique, which samples foci on chromosomes $1,3,6$, and 8 is now available as a commercial test in North America [13, 14]. A gene expression profile test based on RNA has been available as a commercial test, grouping patients into a Class 1 gene expression profile (good prognosis) and a Class 2 gene expression profile (poor prognosis) $[15,16]$. Both DNA- and RNA-based tests are considered to be acceptable platforms for determining a patient's metastatic prognosis when combined with clinical risk factors.

There is the assumption that a good prognosis is conferred for the patient who has a disomy 3 or a Class 1 result with molecular prognostic testing, and that a poor prognosis is associated with a M3 or Class 2 result. With several methods available for determining molecular prognosis, we sought to better understand the results of FISH, gene expression profiling (GEP), and MLPA testing in the same patient. We sought to determine how similar the prognostic result of GEP testing was compared to FISH, and GEP testing was compared to MLPA.

\section{Materials and Methods}

From November 1, 2012, to December 31, 2014, all patients who had been diagnosed with uveal melanoma, treated with brachytherapy, and who underwent intraoperative FNAB (intraoperative 30-gauge transscleral or 27-gauge transvitreal) for molecular prognostication were identified, and the cases were retrospectively reviewed. Only posterior uveal melanoma patients with both GEP and FISH or MLPA molecular test results were included. Patients with iris melanomas were excluded. FNAB technique consisted of a transscleral approach immediately after the anterior tumor border was marked on the sclera using transpupillary transillumination. Multiple passes (numbering between 4 and 7) were taken using a separate 30 -gauge needle per pass. The needle was connected to tubing and attached to a $10-\mathrm{mL}$ syringe, allowing an assistant to create suction while the needle was in the tumor. The assistant collected samples for cytopathology, cytogenetics, GEP, MLPA, and tissue banking. If the assistant did not retrieve an adequate biopsy specimen, an additional pass was performed. Additional maneuvers such as creating a scleral flap, suturing, or cryotherapy were not performed.

Comparison of GEP, M3, and MLPA of

FNAB Specimens in Uveal Melanoma
Cytogenetic testing for M3 on biopsy samples had been performed by FISH at our center since December 2004 and continued until June 2014 when the more accurate MLPA test became commercially available. After November 1, 2014, our center initiated GEP testing with the DecisionDx-UM (Castle Biosciences, Phoenix, AZ, USA) test on biopsy samples, together with FISH testing for M3 in patients undergoing treatment for uveal melanoma. MPLA testing (Impact Genetics, Bowmanville, ON, Canada) became available and was offered to all patients from June 1,2014, to December 31, 2014, together with GEP testing, but FISH testing was not offered during this time due to the known improved accuracy of identifying M3 by MLPA.

Therefore, we examined 2 cohorts of patients: Cohort 1 consisted of patients who underwent intraoperative biopsy for molecular prognosis during brachytherapy with GEP and FISH (November 1, 2012, to January 31, 2014); Cohort 2 consisted of patients who underwent intraoperative biopsy for molecular prognosis during brachytherapy with GEP and MLPA (June 1, 2014, to December 31, 2014). Patient demographics and tumor characteristics were reviewed. The results of biopsy specimens, analyzed by cytopathology, FISH for M3 and 6p gain, GEP analysis with the DecisionDx-UM assay, and MLPA with the Impact Genetics assay were reviewed. A patient was excluded from their cohort if one of the molecular tests failed to amplify. Patient and tumor characteristics and the molecular prognostic test result from tumor biopsies were recorded. 'Concordance' between molecular prognostic tests was defined as when both Class 1 (either Class $1 \mathrm{~A}$ or Class 1B) and disomy 3 were recorded in the same patient, or when Class 2 and M3 were recorded in the same patient. 'Discordance' between tests was defined as when Class 1 (either Class 1A or Class 1B) was found with M3, or when Class 2 was found with disomy 3 in the same patient.

Microsoft Excel (Mircosoft Inc., Redmond, WA, USA) was used to calculate descriptive statistics and also to perform a twotailed t-test to evaluate significance of patient age, tumor height, and ciliary body involvement between concordant and discordant cases in both cohorts.

\section{Results}

A total of 153 consecutive intraocular tumors underwent brachytherapy with intraoperative biopsy, including 141 choroidal melanomas from November 1, 2012, to December 31, 2014. Two cohorts of patients were identified based on prognostic assay availability.

\section{Cohort 1 - Patients with Results from GEP and FISH for M3}

Cohort 1 consisted of 44 patients diagnosed and treated for uveal melanoma in which intraoperative biopsy was performed for molecular prognosis with both GEP and FISH for M3. There were no test failures in this cohort. Patient and tumor characteristics are detailed in Table 1. Concordance and discordance of biopsy results in this cohort of patients are depicted in Figure 1. The over-

Ocul Oncol Pathol 2018;4:16-20 


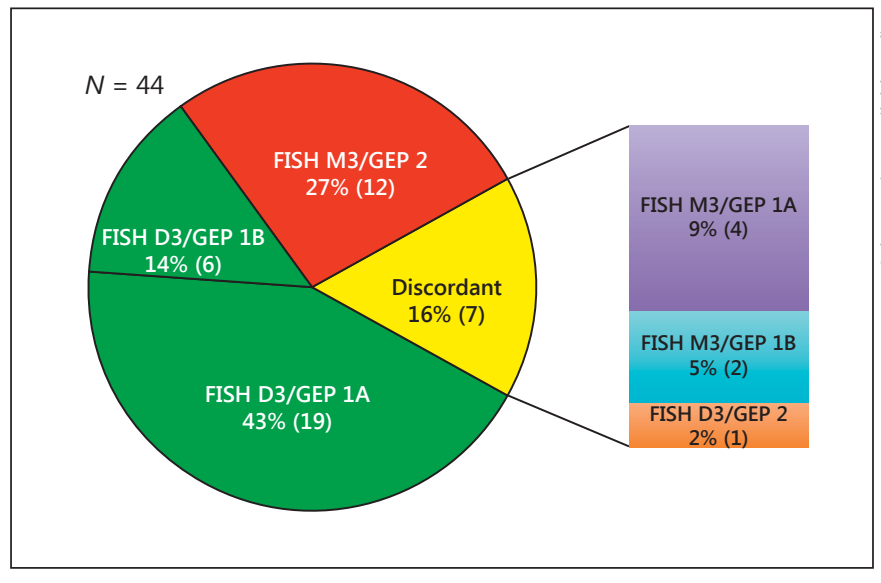

Fig. 1. Concordance of gene expression profiling (GEP) and fluorescent in situ hybridization (FISH). M3, monosomy 3.

Table 1. Cohort 1: DecisionDx-UM GEP versus FISH M3

Choroidal melanoma, $N$

Mean patient age, years

Male

Mean tumor height

Mean tumor basal diameter

Mean follow-up

GEP, gene expression profiling; FISH, fluorescent in situ hybridization; M3, monosomy 3; SD, standard deviation. all concordance of GEP and FISH for M3 was $84.1 \%$. The overall discordance was 15.9\%. Among Class 1 cases, FISH identified $19.3 \%$ ( 6 in 31 ) as having M3, and among Class 2 cases, FISH identified 7.7\% (1 in 13) as having disomy 3 . There was no statistically significant difference between discordant and concordant cases when comparing tumor height $(p=0.79)$, patient age $(p=0.57)$, and ciliary body involvement $(p=0.40)$.

\section{Cohort 2 - Patients with Results from GEP and MLPA} for M3

Cohort 2 consisted of 49 patients diagnosed and treated for uveal melanoma in whom intraoperative biopsy was performed for molecular prognosis using both GEP and MLPA for M3. Six patients were excluded due to test failures (3 MLPA samples, 3 GEP samples), resulting in a total of 43 patients in Cohort 2. Patient and tumor characteristics are detailed in Table 2. Concordance and discordance of biopsy results in this cohort of patients is de-

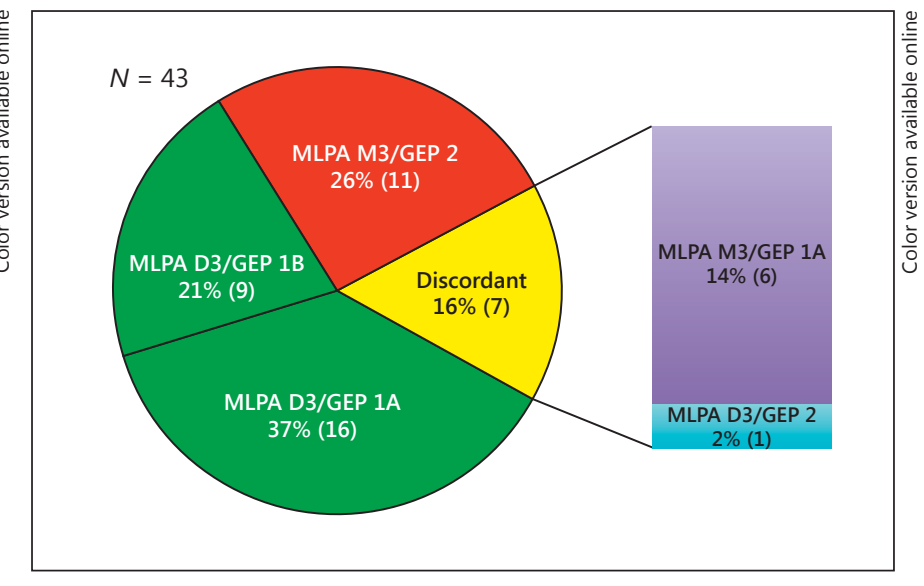

Fig. 2. Concordance of gene expression profiling (GEP) and multiplex ligation probe amplification (MLPA). M3, monosomy 3.

Table 2. Cohort 2: Decision Dx-UM GEP versus MLPA (Impact Genetics, Bowmanville, ON, Canada)

\begin{tabular}{lc}
\hline Choroidal melanoma, $N$ & 43 \\
Mean patient age, years & $61($ range $29-80)$ \\
Male & $47 \%(n=20)$ \\
Mean tumor height & $4.54 \mathrm{~mm}(\mathrm{SD}=2.85)$ \\
Mean tumor basal diameter & $12.33 \mathrm{~mm}(\mathrm{SD}=3.42)$ \\
Mean follow-up & 8.9 months (range 5.4-11.4) \\
\hline
\end{tabular}

GEP, gene expression profiling; MLPA, multiplex ligation probe amplification; SD, standard deviation.

picted in Figure 2. The overall concordance of GEP and MLPA for M3 was $83.7 \%$. The overall discordance was 16.3\%. Among Class 1 cases, MLPA identified 19.3\% (6 in 31) as having M3, and among Class 2 cases, MLPA identified $8.3 \%$ ( 1 in 12 ) as having disomy 3 . There was no statistically significant difference between discordant and concordant cases when comparing tumor height $(p=$ $0.50)$ or patient age $(p=0.055)$.

There were no cases of confirmed metastatic disease in either cohort during the limited follow-up period of this study (cohort 1 - mean 22.2 months, range 15.9-29.9; cohort 2 - mean 8.9 months, range 5.4-11.4).

\section{Discussion}

We have demonstrated that there is an approximately $84 \%$ agreement rate in molecular prognosis between FISH testing and GEP, and between MLPA testing and
18

Ocul Oncol Pathol 2018;4:16-20 DOI: $10.1159 / 000468941$
Klufas/Richter/Itty/Moreno/McCannel/ McCannel 
GEP. The highest rates of discordance were found among Class 1 cases in both FISH/GEP and MLPA/GEP at 19.3\%. This essentially meant that when GEP was used in conjunction with MLPA or FISH, about 1 in 5 patients with Class 1 tumors were identified as M3. Tumor height was not significantly different between concordant and discordant groups in both cohorts.

Molecular testing for prognosis in patients with uveal melanoma has become standard of care in the management of primary tumors. Studies have demonstrated that patients want to know their prognosis with respect to the development of metastasis, whether or not there are effective treatments for metastatic disease [17]. It has been suggested that information and less uncertainty may empower patients and help them better address aspects of their cancer and how it affects their life. The two main methods of molecular testing involve determining M3 status of the tumor $[9,10,14]$, one is a DNA-based approach, and the other makes use of a commercially available RNA-based GEP test that groups patients into Class 1 (low risk for metastasis) and Class 2 (high risk for metastasis) $[15,18]$. The literature supports the use of both tests as good predictors of metastatic outcome [19].

However, we have shown in this paper that what constitutes 'good prognosis', that is a disomy 3 or a Class 1 test result, does not necessarily correlate when tumor biopsy samples are taken from the same patient. In fact, both FISH testing and MLPA testing to determine the status of chromosome 3 within the tumor have a 15 and $16 \%$ rate of disagreement or discordance, respectively, when compared to GEP testing, thereby identifying more patients with a poor prognosis than with GEP, with MLPA being more sensitive than FISH in determining chromosome 3 status, which we and others have reported previously. Our results are similar to a report by Onken et al. [20], where 260 patients with uveal melanoma in their series underwent both GEP testing and a single-nucleotide polymorphism test (developed by the authors) for determining M3 status. There was a similar discordance rate of $14.6 \%$ (38 patients) where prognostic biopsy resulted in a Class 1 result and a M3 result. Regarding those patients with Class 2 and disomy 3 results, there was a $6.2 \%$ discordance rate in their study, compared to our $3.8 \%$ with FISH and $3.1 \%$ with MLPA.

It is not entirely clear why a consistent percentage of at least $15 \%$ of the patients (19\% when MLPA was used) with a Class 1 test result should also have a M3 test result in our series as well as in the larger series by Onken et al. [20]. Although the published literature supports a good prognosis in the short-term, it is well known that Class 1 patients may

Comparison of GEP, M3, and MLPA of FNAB Specimens in Uveal Melanoma indeed develop metastasis and that the percentage of metastasizing Class 1 melanomas increases with longer follow-up than initial reports indicated. Perhaps DNA testing is more sensitive at detecting high risk for metastasis patients. RNA and its inherent instability compared to DNA may be more prone to handling error, and perhaps this may be the source of an inherently inaccurate test in the setting of GEP. It has also been suggested that the size of the tumor must be considered when interpreting a Class 1 or a Class 2 test result [21]. However, we found that the average tumor size was not statistically different between concordant and discordant groups of patients in our series.

Tumor heterogeneity may possibly account for the discordant test results [1,22-25]. Each biopsy sample was obtained by an independent needle pass. Therefore, a separate area of the tumor with potentially different molecular make-up may have been sampled. However, the consistency of the discordance rate of $15 \%$ (patients with Class 1 and M3) suggests that the Class1/M3 patients may be a different and unique subset whose test results may not be entirely attributable to random heterogeneity.

Although there are no long-term published reports on the rate of Class 1 uveal melanomas that ultimately metastasize, there are many anecdotal instances of patients initially diagnosed with a low-risk uveal melanoma that went on to develop liver metastasis. There are also instances of disomy 3 uveal melanoma that have metastasized. With MLPA and microsatellite techniques, and improved DNAbased testing over simple FISH testing, karyotype and $\mathrm{CGH}$, cases of isodisomy 3 that can mistakenly give a good prognosis test with these methods, can now be detected resulting in a high risk for metastasis. Furthermore, the commercial MLPA test also sequences for a GNAQ or GNA11 mutation in cases of disomy 3 to help confirm that actual uveal melanoma tissue has been submitted for analysis. GNA mutations have been reported in $85-91 \%$ of all uveal melanomas. With GEP testing, we and others have reported Class 1 and Class 2 test results when nonuveal melanoma tissue is sampled $[26,27]$. Some degree of testing inaccuracy on the part of the commercially available gene expression profile test may also contribute to the more sensitive detection of M3 in DNA-based approaches.

It is important to keep in mind that metastatic prognosis is a multifactorial assessment [7]. One must exercise caution with a molecular test result alone. Using a multivariate analysis, Damato et al. [28] have established a robust database that makes use of not only chromosome 3 status but also patient and tumor clinical factors such as age, tumor size, and also other histological characteristics when available to determine prognosis.

Ocul Oncol Pathol 2018;4:16-20 DOI: $10.1159 / 000468941$ 
Long-term studies are necessary to determine the true accuracy of available molecular testing platforms for prognosis, as metastatic disease becomes manifest in patients. At this point, however, there is sufficient data to use all prognostic testing methods (including clinical features alone) to help counsel patients about their cancer and systemic surveillance. Continued follow-up and evaluation of existing prognostic testing methods including future ones must be continuously studied.

\section{Acknowledgments}

This work was supported by the George E. and Ruth Moss Trust and an unrestricted grant from the Research to Prevent Blindness.

\section{Statement of Ethics}

The study was performed in accordance with the United States Health Insurance Portability and Accountability Act (HIPAA) of 1996 and was approved by the Office of the Human Research Protection Program (Institutional Review Board) of the University of California, Los Angeles, CA, USA.

\section{Disclosure Statement}

Dr. Tara McCannel is an uncompensated member of the Advisory Board of Impact Genetics. All other authors report no relevant conflicts of interest.

\section{References}

1 Correa ZM: Assessing prognosis in uveal melanoma. Cancer Control 2016;23:93-98.

2 Damato B: Progress in the management of patients with uveal melanoma. The 2012 Ashton Lecture. Eye (Lond) 2012;26:1157-1172.

3 Field MG, Harbour JW: Recent developments in prognostic and predictive testing in uveal melanoma. Curr Opin Ophthalmol 2014;25: 234-239.

4 Hamadeh F, Medina CA, Singh AD, Reynolds JP, Biscotti CV: Uveal melanoma: an analysis of cellular features and comparison to monosomy 3 status. Diagn Cytopathol 2016;44: 377-383.

5 McCannel TA: Fine-needle aspiration biopsy in the management of choroidal melanoma. Curr Opin Ophthalmol 2013;24:262-266.

6 Shields CL, Ganguly A, Bianciotto CG, Turaka K, Tavallali A, Shields JA: Prognosis of uveal melanoma in 500 cases using genetic testing of fine-needle aspiration biopsy specimens. Ophthalmology 2011;118:396-401.

7 Singh AD: Prognostication of uveal melanoma: a work in progress. JAMA Ophthalmol 2016;134:740-741.

8 Singh AD, Medina CA, Singh N, Aronow ME, Biscotti CV, Triozzi PL: Fine-needle aspiration biopsy of uveal melanoma: outcomes and complications. Br J Ophthalmol 2016;100: 456-462.

9 Prescher G, Bornfeld N, Hirche H, Horsthemke B, Jockel KH, Becher R: Prognostic implications of monosomy 3 in uveal melanoma. Lancet 1996;347:1222-1225.

10 Tschentscher F, Husing J, Holter T, et al: Tumor classification based on gene expression profiling shows that uveal melanomas with and without monosomy 3 represent two distinct entities. Cancer Res 2003;63:25782584.

11 Tschentscher F, Prescher G, Horsman DE, et al: Partial deletions of the long and short arm of chromosome 3 point to two tumor suppressor genes in uveal melanoma. Cancer Res 2001;61:3439-3442.

12 Young TA, Rao NP, Glasgow BJ, Moral JN, Straatsma BR: Fluorescent in situ hybridization for monosomy 3 via 30 -gauge fine-needle aspiration biopsy of choroidal melanoma in vivo. Ophthalmology 2007;114:142-146.

13 Damato B, Dopierala J, Klaasen A, van Dijk M, Sibbring J, Coupland SE: Multiplex ligation-dependent probe amplification of uveal melanoma: correlation with metastatic death. Invest Ophthalmol Vis Sci 2009;50:30483055.

14 Damato B, Dopierala JA, Coupland SE: Genotypic profiling of 452 choroidal melanomas with multiplex ligation-dependent probe amplification. Clin Cancer Res 2010;16:60836092.

15 Harbour JW, Chen R: The DecisionDx-UM gene expression profile test provides risk stratification and individualized patient care in uveal melanoma. PLoS Curr 2013;5.

16 Onken MD, Worley LA, Ehlers JP, Harbour JW: Gene expression profiling in uveal melanoma reveals two molecular classes and predicts metastatic death. Cancer Res 2004;64: 7205-7209.

17 Beran TM, McCannel TA, Stanton AL, Straatsma BR, Burgess BL: Reactions to and desire for prognostic testing in choroidal melanoma patients. J Genet Couns 2009;18:265274.

18 Onken MD, Worley LA, Tuscan MD, Harbour JW: An accurate, clinically feasible multi-gene expression assay for predicting metastasis in uveal melanoma. J Mol Diagn 2010;12:461-468.

19 Werdich XQ, Jakobiec FA, Singh AD, Kim IK: A review of advanced genetic testing for clinical prognostication in uveal melanoma. Semin Ophthalmol 2013;28:361-371.
20 Onken MD, Worley LA, Char DH, et al: Collaborative Ocular Oncology Group report number 1: prospective validation of a multigene prognostic assay in uveal melanoma. Ophthalmology 2012;119:1596-1603.

21 Walter SD, Chao DL, Feuer W, Schiffman J, Char DH, Harbour JW: Prognostic implications of tumor diameter in association with gene expression profile for uveal melanoma. JAMA Ophthalmol 2016;134:734-740.

22 Chang MY, Rao NP, Burgess BL, Johnson L, McCannel TA: Heterogeneity of monosomy 3 in fine needle aspiration biopsy of choroidal melanoma. Mol Vis 2013;19:1892-1900.

23 Coupland SE, Kalirai H, Ho V, Thornton S, Damato BE, Heimann H: Concordant chromosome 3 results in paired choroidal melanoma biopsies and subsequent tumour resection specimens. Br J Ophthalmol 2015;99: 1444-1450.

24 Damato B, Duke C, Coupland SE, et al: Cytogenetics of uveal melanoma: a 7-year clinical experience. Ophthalmology 2007;114:1925-1931.

25 Dopierala J, Damato BE, Lake SL, Taktak AF, Coupland SE: Genetic heterogeneity in uveal melanoma assessed by multiplex ligation-dependent probe amplification. Invest Ophthalmol Vis Sci 2010;51:4898-4905.

26 Klufas MA, Itty S, McCannel CA, Glasgow BJ, Moreno C, McCannel TA: Variable results for uveal melanoma-specific gene expression profile prognostic test in choroidal metastasis. JAMA Ophthalmol 2015;133:1073-1076.

27 Seider MI, Stewart PJ, Mishra KK, Damato BE: Uveal melanoma gene expression profile test result provided for uveal metastasis. Ophthalmic Surg Lasers Imaging Retina 2014;45: 441-442.

28 Damato B, Eleuteri A, Taktak AF, Coupland SE: Estimating prognosis for survival after treatment of choroidal melanoma. Prog Retin Eye Res 2011;30:285-295. 